\title{
The Design of Heart Rate Detector and Body Temperature Measurement Device Using ATMega16
}

\author{
Nur Hudha Wijaya ${ }^{1}$, Fajar Ahmad Fauzi ${ }^{2}$, Elsayed T.Helmy ${ }^{3}$, Phong Thanh Nguyen ${ }^{4}$, Rachmad Andri Atmoko \\ ${ }^{1,2}$ Department of Electrical Engineering, Universitas Muhammadiyah Yogyakarta, Yogyakarta, Indonesia \\ ${ }^{3}$ National Institute of oceanography and fisheries, Alexandria, Egypt \\ ${ }^{4}$ Director, Department of Project Management, Ho Chi Minh city Open University, Vietnam \\ ${ }^{5}$ School Of Mechanical and Electrical Engineering, Guilin University of Electronic Technology, China \\ hudha@umy.ac.id ${ }^{1}$, fajar.ahmad.2013@vokasi.umy.ac.id ${ }^{2}$, stalaat41@gmail.com ${ }^{3}$, phong.nt@ ou.edu.vn ${ }^{4}$, \\ mokoraden@hotmail.com ${ }^{5}$
}

\begin{abstract}
The research discussed health services in the role of diagnostics and life support. The designed system was a system able to provide information on the user's health condition, in this case, a measure of heart rate and body temperature using a heart rate sensor (finger-tip sensor). It took the data from the blood flow on the index finger for 60 seconds, then displayed it on the LCD. The LM35 temperature sensor was used for body temperature data collection. Changes in the sensor heat would be converted into electricity, translated into digital form through a 10-bit ADC, processed by the ATMega 16 microcontroller, and displayed on the LCD. The results showed that the error of each parameter of heart rate and body temperature was $<1.702 \%$ and $<0.55 \%$.
\end{abstract}

\section{Keywords - finger-tip, body temperature, microcontroller}

\section{INTRODUCTION}

Physical health is measured from the basic parameters of normal values of the body's vital signs, including heart rate and body temperature. The heart is the most important organ in the human body because it is the main organ to circulate blood throughout the body. People usually utilize a stethoscope and thermometer to measure them. In fact, because of the high price but less practical, few people use such devices. Hence, a device that can test the body's state in a practical and portable way is required.

Some researchers have researched on temperature sensors and microcontrollers such as Yilei and Airong who designed a real-time temperature monitoring based on the operating system [1]. The system consisted of a DS18B20 sensor, an nRF24L01 wireless module, and a STM32F103ZET6 microcontroller. Fuady et al studied extreme machine learning and neural network to control the temperature and humidity of oysters based on microcontrollers [2]. The system consisted of a microcontroller and a DHT 11 sensor. Wang and Chi investigated the Arduino Uno platform wireless temperature and humidity monitoring system consisting of an Arduino microcontroller, a temperature sensor and humidity sensor, a $128641 \mathrm{LCD}$, and an nRF24L0 wireless module [3].
Other temperature measurement research includes an experiment to precise temperature measurement using platinum RTD PT1000 temperature sensor and Matlab by Chauhan and Neelakantan [4], a unit design for measuring the temperature of moving parts of the crank mechanism by Elkhutov using a temperature sensor and accelerometer [5], and Arduino-based temperature and humidity controls for condensation on engineered surface wettability by Gupta et al using a temperature sensor, a humidity sensor, and an Arduino Uno microcontroller [6].

Some other researchers have conducted research on body temperature. Saha, Raun, and Saha monitored the health of patients with smart ambulance systems using the Internet of Things (IOTs) [7]. The system consisted of a heart rate sensor, a temperature sensor, a microcontroller using Arduino, and a Raspberry Pi. Priyadharshini developed an embedded web server for health care systems using E-cards consisting of a heart rate sensor, a blood sugar sensor, a blood pressure sensor, a weight sensor, and an ATMEGA16 microcontroller [8]. Jayswal, Gupta and Gupta studied patient health monitoring systems based on the Internet of Things including a heart rate sensor, a temperature sensor, an ATMEGA328 microcontroller, and a Wi-Fi module [9].

Asmidar et al developed infant incubators for clinics in rural areas of Malaysia [10]. The system consisted of an ATMEGA2560 microcontroller, a DHT11 incubator temperature and humidity sensor, a DS18B20 baby body temperature sensor, a weight sensor, and an LM35 heating element temperature sensor. Sudha et al designed a system for patient monitoring in hospital management using $\mathrm{Li}-\mathrm{Fi}$ [11]. The system consisted of a heart rate sensor, a blood sugar sensor, a temperature sensor, a respiratory sensor, a PIC 16F844 microcontroller, and a LIFI transmitter. Aziz et al developed a smart real-time health monitoring and tracking system using GSM/GPS technology that included a GPS position sensor, a microcontroller, a pulse sensor, a temperature sensor, and a GSM module [12]. Ganesh et al designed low-cost smart chairs for telemedicine and IoT- 
based health monitoring. It was an open-source technology to facilitate better health care [13]. The system consisted of a blood pressure sensor, a temperature sensor, a weight sensor, an analog signal processing module, microcontrollers, a GSM module, a Wi-Fi module, and a Bluetooth module. Dhande et al developed portable systems for monitoring vital signs with a Java-based computer display and recording of data that consisted of a heart rate sensor, a temperature sensor, a microcontroller, and a laptop [14]. Thomas et al examined sensing heart rate and body temperature digitally including an Arduino Uno microcontroller, a temperature sensor, a heart rate sensor, and a Bluetooth module [15].

Other research on body temperature measurements include body temperature measurements for remote health monitoring systems consisting of a temperature sensor, microcontrollers, and Zigbee by Mansor et al [16], continuous heart rate and body temperature monitoring systems using Arduino UNO and Android devices containing a temperature sensor, heart rate sensor, Arduino, low pass filter module and Bluetooth module by Asaduzzaman Miah et al [17], and design and implementation of smart health bands for measurement of blood pressure, pulse, and body temperature by Rahman, Islam and Ahmad [18].

Kioumars and Tang studied wireless networks for health monitoring, using heart rate and temperature sensors [19]. The system consisted of an Arduino microcontroller, a TMP36 temperature sensor, a heart rate sensor, and a wireless communication module. Gulcharan et al investigated the stability and reliability of the patient's wireless temperature monitoring devices using a temperature sensor, an Arduino microcontroller, and a Zigbee [20]. Rahman et al developed a device for remote monitoring of heart rate and body temperature using a heart rate sensor, temperature sensor, Ethernet host, web server, and a microcontroller [21].

Heart rate monitoring can be done indirectly by utilizing blood vessels, namely, by conducting leads or sensors on the blood flow. The first-time blood from a vein enters the Right Atrium, then goes to the Right Ventricle, next to the Lungs, wherein the lungs the air exchange from $\mathrm{CO} 2$ to $\mathrm{O} 2$. From the lungs, the blood goes to the Left Atrium, then to the Left Ventricle, and the entire body and head through the Aorta veins. The research aims to measure heart rate and body temperature using a heart rate sensor (finger-tip sensor), an LM35 temperature sensor, and an ATMega16 microcontroller.

\section{METHOD}

The device was expected to meet the specification, namely, to count the number of heartbeats in one minute, and to measure body temperature constantly. Figure 1 displays the block diagram of the system. It shows that one of the fingers is inserted into the finger sensor transducer block, then the LED light that penetrates the finger will be received by the LDR where the frequency of the blood flow will be detected. The data will be processed on the microcontroller, and the results will be displayed on seven segments that show the number of hearts beats every minute [21] - [25].

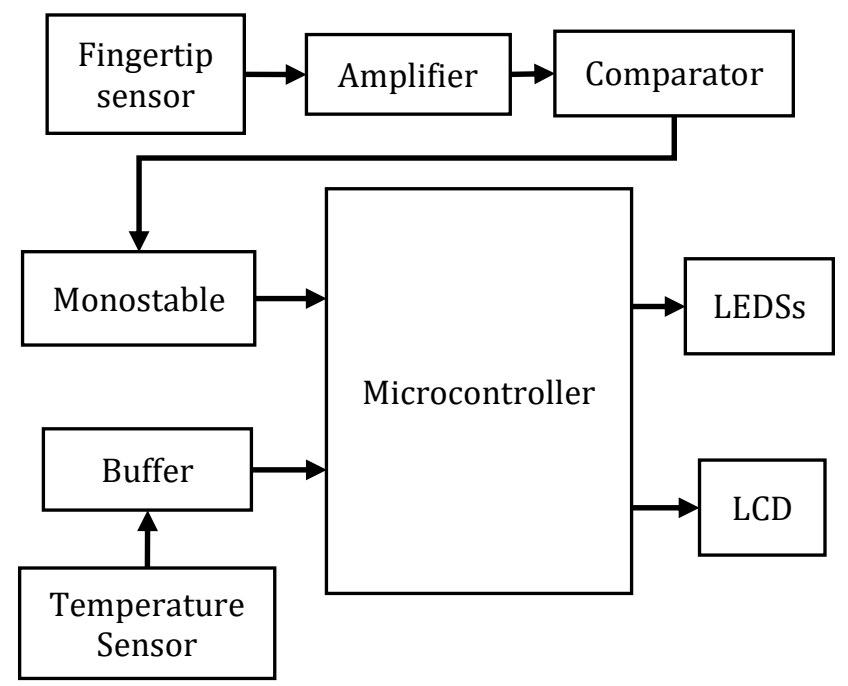

Fig. 1. System block diagram

\section{a. Hardware design}

In the hardware design, the system was divided into important parts, namely the Heart Rate Sensor block [20], LM35 Temperature Sensor [25]-[30], Main Controller (ATMega16), LED, LCD, and Power Supply.

Figure 2 displays the heart rate sensor schematic. This series of blocks work as a detector of the heart rate through the fingers. This sensor circuit is inserted via PORTB.0 on the microcontroller. Figure 3 illustrates the temperature sensor schematic.

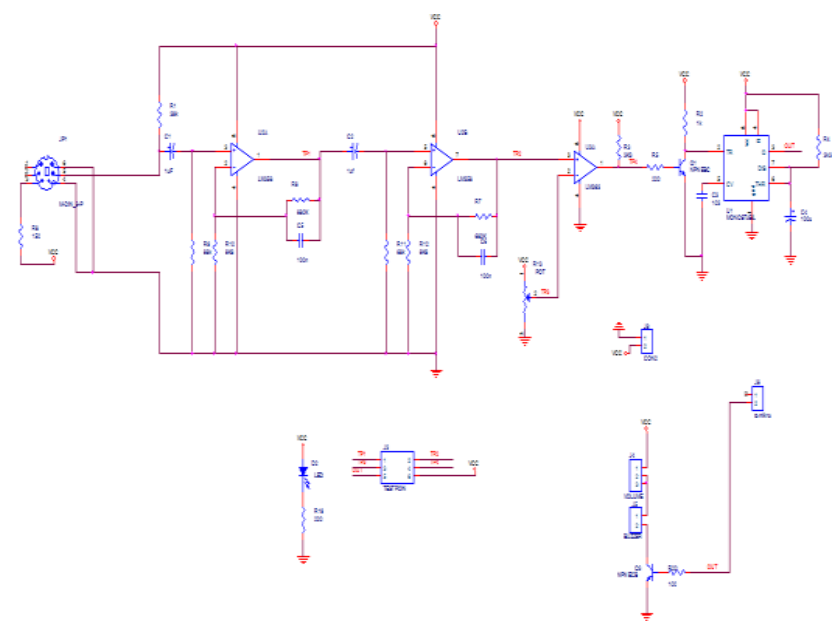

Fig. 2. Heart rate sensor schematic

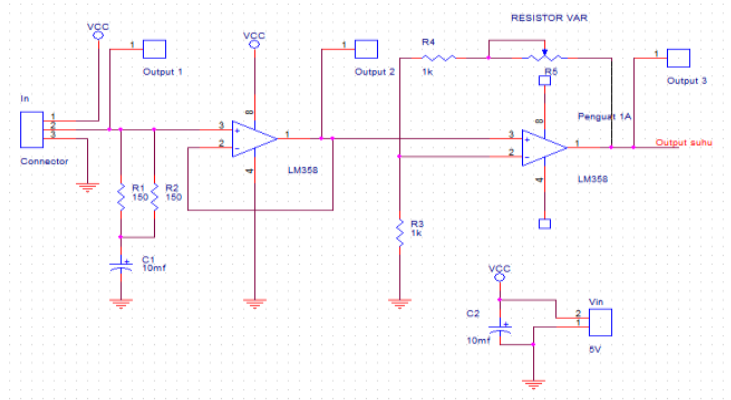

Fig. 3. Temperature sensor schematic 
Based on the Figure 3, the temperature sensor works by utilizing changes in temperature converted into electrical signals. The IC ATMega 16 serves as the main control in processing data. In the application of the system, the PORTB.0 is as the input from BPM (Heart Rate Gauge) and PORTA.0 as the temperature (Temperature Gauge) input. The main system schematic was shown in Figure 4.

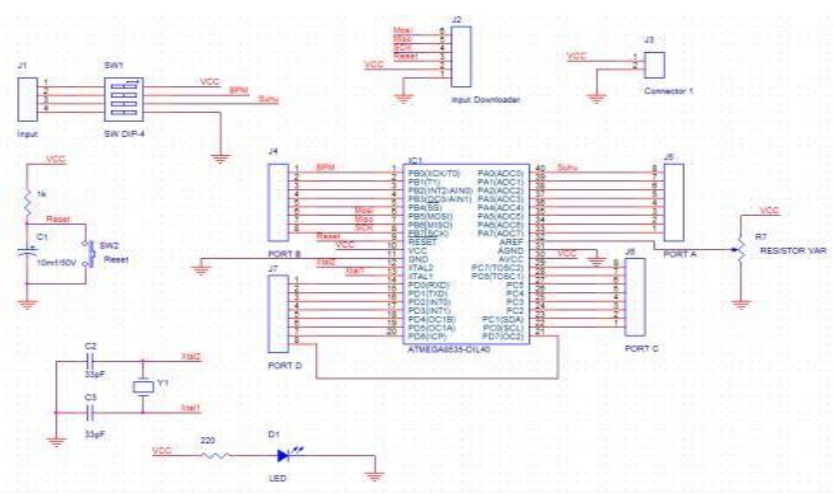

Fig. 4. Example of a figure caption.

\section{b. Firmware design}

The workflow of the system illustrated in Figure 5 shows that the sensor starts to work (counting the number of the heartbeats and body temperature) when the start button is activated. The reset button works to repeat the counting from the beginning.

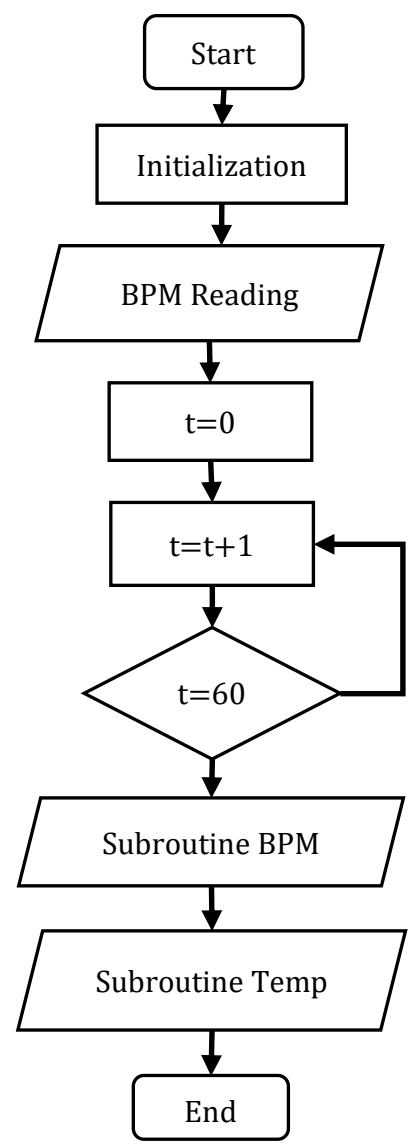

Fig. 5. System workflow

\section{SYSTEM TEST}

\section{a. Heart rate measurement}

In the heart rate measurement test, the device calculated the frequency of blood flow flowing of two patients for 60 seconds five times. The other device used as a comparison was Mindray Oximeter. The results of the test were presented in Table 1.

TABLE I. HEART RATE MEASUREMENT RESULT

\begin{tabular}{|c|c|c|c|c|}
\hline \multirow{2}{*}{$\begin{array}{c}\text { No. } \\
\text { measurement }\end{array}$} & \multicolumn{2}{|c|}{ Patient 1 } & \multicolumn{2}{c|}{ Patient 2 } \\
\cline { 2 - 5 } & $\begin{array}{c}\text { Tested } \\
(\mathrm{BPM})\end{array}$ & $\begin{array}{c}\text { Standart } \\
(\mathrm{BPM})\end{array}$ & $\begin{array}{c}\text { Tested } \\
(\mathrm{BPM})\end{array}$ & $\begin{array}{c}\text { Standart } \\
(\mathrm{BPM})\end{array}$ \\
\hline 1 & 77 & 80 & 71 & 70 \\
\hline 2 & 72 & 76 & 75 & 73 \\
\hline 3 & 73 & 76 & 71 & 71 \\
\hline 4 & 73 & 74 & 71 & 73 \\
\hline 5 & 75 & 76 & 72 & 73 \\
\hline Mean & 74 & 76.4 & 72 & 72 \\
\hline Standard deviation & 2.7 & \multicolumn{2}{|c|}{0.6} \\
\hline UA & & $1.2 \%$ & \multicolumn{2}{|c|}{0} \\
\hline \% error & & & \multicolumn{3}{|c|}{} \\
\hline
\end{tabular}

\section{b. Body temperature measurement}

Table 2 present the body temperature measurement results by using the tested device and a thermometer as the comparator.

TABLE II. BODY TEMPERATURE MEASUREMENT RESULT

\begin{tabular}{|c|c|c|}
\hline \multirow{2}{*}{$\begin{array}{c}\text { No. } \\
\text { measurement }\end{array}$} & $\begin{array}{c}\text { Measurement device } \\
\text { Tested }\end{array}$ & $\begin{array}{c}\text { Standard } \\
\text { (BPM) }\end{array}$ \\
\cline { 2 - 3 } & 36 & 36 \\
\hline 2 & 36 & 37 \\
\hline 3 & 36 & 36 \\
\hline 4 & 36 & 36 \\
\hline 5 & 36 & 36 \\
\hline Mean & 36 & 0.4 \\
\hline Standard deviation & $0.2 \%$ \\
\hline UA & & 0.6 \\
\hline \% error & & \\
\hline
\end{tabular}

\section{CONCLUSIONS}

The error value of the LM35 sensor measurement compared to the thermometer is $1.903 \%$. This sensor is very sensitive to vibration and light. If one pulse is lost in monitoring, it will cause a loss of six beats counting. The error of the BPM measurement compared to the calibration devices $0.55 \%$.

\section{REFERENCES}

[1] F. Yilei and W. Airong, "The design of real-time temperature monitoring based on embedded operating system," in 2017 Chinese Automation Congress (CAC), 2017, vol. 2017-Janua, pp. 34003403 . 
[2] G. M. Fuady et al., "Extreme learning machine and back propagation neural network comparison for temperature and humidity control of oyster mushroom based on microcontroller," in 2017 International Symposium on Electronics and Smart Devices (ISESD), 2017, vol. 2018-Janua, no. 1, pp. 46-50.

[3] Y. Wang and Z. Chi, "System of Wireless Temperature and Humidity Monitoring Based on Arduino Uno Platform," in 2016 Sixth International Conference on Instrumentation \& Measurement, Computer, Communication and Control (IMCCC), 2016, pp. 770773 .

[4] J. Chauhan and U. Neelakantan, "An experimental approach for precise temperature measurement using platinum RTD PT1000," in 2016 International Conference on Electrical, Electronics, and Optimization Techniques (ICEEOT), 2016, pp. 3213-3215.

[5] S. Elkhutov, "Unit for Measuring the Temperature of Moving Parts of the Crank Mechanism," in 2018 XIV International ScientificTechnical Conference on Actual Problems of Electronics Instrument Engineering (APEIE), 2018, pp. 39-41.

[6] R. Gupta, C. Das, A. Roy, R. Ganguly, and A. Datta, “Arduino based temperature and humidity control for condensation on wettability engineered surfaces," in 2018 Emerging Trends in Electronic Devices and Computational Techniques (EDCT), 2018, pp. 1-6.

[7] H. N. Saha, N. F. Raun, and M. Saha, "Monitoring patient's health with smart ambulance system using Internet of Things (IOTs)," in 2017 8th Annual Industrial Automation and Electromechanical Engineering Conference (IEMECON), 2017, pp. 91-95.

[8] J. Priyadharshini, "Development of embedded web server for health care system using E-card," in 2017 International Conference on Algorithms, Methodology, Models and Applications in Emerging Technologies (ICAMMAET), 2017, vol. 2017-Janua, pp. 1-5.

[9] R. Jayswal, R. Gupta, and K. K. Gupta, "Patient health monitoring system based on Internet of Things," in 2017 Fourth International Conference on Image Information Processing (ICIIP), 2017, pp. 14.

[10] N. Asmidar, N. Binti, M. Fudzi, and N. Binti, "Development of infant incubator for clinic in the rural area of Malaysia," in 2016 IEEE EMBS Conference on Biomedical Engineering and Sciences (IECBES), 2016, pp. 331-334.

[11] S. Sudha, D. Indumathy, A. Lavanya, M. Nishanthi, D. M. Sheeba, and V. Anand, "Patient monitoring in the hospital management using Li-Fi," in 2016 IEEE Technological Innovations in ICT for Agriculture and Rural Development (TIAR), 2016, no. Tiar, pp. 9396.

[12] K. Aziz, S. Tarapiah, S. H. Ismail, and S. Atalla, "Smart real-time healthcare monitoring and tracking system using GSM/GPS technologies," in 2016 3rd MEC International Conference on Big Data and Smart City (ICBDSC), 2016, pp. 1-7.

[13] G. R. D. Ganesh, K. Jaidurgamohan, V. Srinu, C. R. Kancharla, and S. V. S. Suresh, "Design of a low cost smart chair for telemedicine and IoT based health monitoring: An open source technology to facilitate better healthcare," in 2016 11th International Conference on Industrial and Information Systems (ICIIS), 2016, pp. 89-94.

[14] O. S. Dhande, R. R. Nambiar, Y. R. Deshpande, and P. Dhage, "Portable system to monitor vital signs with a Java based computer display and data logging," in 2016 Conference on Advances in Signal Processing (CASP), 2016, no. Fig 1, pp. 144-149.

[15] S. S. Thomas, A. Saraswat, A. Shashwat, and V. Bharti, "Sensing heart beat and body temperature digitally using Arduino," in 2016 International Conference on Signal Processing, Communication, Power and Embedded System (SCOPES), 2016, pp. 1721-1724.

[16] H. Mansor, M. H. A. Shukor, S. S. Meskam, N. Q. A. M. Rusli, and N. S. Zamery, "Body temperature measurement for remote health monitoring system," in 2013 IEEE International Conference on Smart Instrumentation, Measurement and Applications (ICSIMA), 2013, no. November, pp. 1-5.

[17] M. Asaduzzaman Miah, Mir Hussain Kabir, M. Siddiqur Rahman Tanveer, and M. A. H. Akhand, "Continuous heart rate and body temperature monitoring system using Arduino UNO and Android device," in 2015 2nd International Conference on Electrical Information and Communication Technologies (EICT), 2015, no. Eict, pp. 183-188.

[18] M. H. Rahman, M. R. Islam, and M. Ahmad, "Design and Implementation of a Smart Health Band for the Measurement of Blood Pressure, Pulse Rate and Body Temperature," in 2018 4th International Conference on Electrical Engineering and Information \& Communication Technology (iCEEiCT), 2018, pp. 156-161.

[19] A. H. Kioumars and L. Tang, "Wireless network for health monitoring: heart rate and temperature sensor," in 2011 Fifth International Conference on Sensing Technology, 2011, pp. 362369.

[20] N. F. B. I. Gulcharan, H. Daud, N. M. Nor, T. Ibrahim, and M. Z. Shamsudin, "Investigation of Stability and Reliability of the Patient's Wireless Temperature Monitoring Device," Procedia Comput. Sci., vol. 40, no. C, pp. 151-159, 2014

[21] M. A. Rahman, A. Barai, M. A. Islam, and M. M. A. Hashem, "Development of a device for remote monitoring of heart rate and body temperature," in 2012 15th International Conference on Computer and Information Technology (ICCIT), 2012, pp. 411416.

[22] C. Cristea, A. Pasarica, G. Andruseac, B. Dionisie, and C. Rotariu, "A wireless ECG acquisition device for remote monitoring of heart rate and arrhythmia detection," in 2015 E-Health and Bioengineering Conference (EHB), 2015, pp. 1-4.

[23] I. H. De Oliveira, V. H. Cene, and A. Balbinot, "Portable electrocardiograph through android application," in 201537 th Annual International Conference of the IEEE Engineering in Medicine and Biology Society (EMBC), 2015, pp. 6780-6783.

[24] R. Umega and M. A. Raja, "Design and implementation of livestock barn monitoring system," in 2017 International Conference on Innovations in Green Energy and Healthcare Technologies (IGEHT), 2017, pp. 1-6.

[25] M. Ghamari, C. Aguilar, C. Soltanpur, and H. Nazeran, "Rapid Prototyping of a Smart Device-Based Wireless Reflectance Photoplethysmograph," in 2016 32nd Southern Biomedical Engineering Conference (SBEC), 2016, pp. 175-176.

[26] N. A. Zakaria, F. N. B. Mohd Saleh, and M. A. A. Razak, "IoT (Internet of Things) Based Infant Body Temperature Monitoring," in 2018 2nd International Conference on BioSignal Analysis, Processing and Systems (ICBAPS), 2018, pp. 148-153.

[27] M. R. Desai and S. Toravi, “A Smart Sensor Interface for Smart Homes and Heart Beat Monitoring using WSN in IoT Environment," in 2017 International Conference on Current Trends in Computer, Electrical, Electronics and Communication (CTCEEC), 2017, pp. 74-77.

[28] Gobhinath S., Aparna V, and Azhagunacchiya R, "An automatic driver drowsiness alert system by using GSM," in 2017 11th International Conference on Intelligent Systems and Control (ISCO), 2017, pp. 125-128.

[29] P. Bagchi et al., "Intelligent health diagnosis and patient guidance system," in 2017 8th IEEE Annual Information Technology, Electronics and Mobile Communication Conference (IEMCON), 2017, pp. 269-272.. 